\title{
A PENDULUM-BASED MODEL FOR FLUID STRUCTURE INTERACTION ANALYSES
}

\author{
D. A. M. Martins ${ }^{a}$, \\ A. Silveira-Neto ${ }^{a}$, \\ and V. Steffen Jr. ${ }^{\text {a }}$ \\ ${ }^{a}$ Universidade Federal de Uberlândia \\ Faculdade de Engenharia Mecânica \\ Campus Santa Mônica - Bloco 1M \\ CEP. 38400-902, Uberlândia, Minas Gerais, \\ Brasil \\ aristeus@mecanica.ufu.br

\begin{abstract}
In the present contribution a pendulum-based model is used to represent fluid-structure interactions. For this purpose, a theoretical and experimental analysis of the dynamic behavior of a viscously damped spherical non rotational single-degree-of-freedom pendulum was performed. The damping process is characterized according to two different regimes: in the first, it can be seen as a viscous low Reynolds' number flow and, in the second, it can be considered as a viscously damped system. The pendulum was tested experimentally in a large reservoir containing a Newtonian fluid for validation purposes and good agreement was found between numerical and experimental results.

Keywords: flow visualization, pendulum, damped system, drag coefficient.
\end{abstract}

\section{NOMENCLATURE}

$A_{p} \quad$ area of the sphere projected on a plane normal to the direction of its displacement, $\mathrm{m}^{2}$

$\mathrm{a}_{\mathrm{t}}$ acceleration in the tangential direction, $\mathrm{m} / \mathrm{s}^{2}$

B buoyancy, $\mathrm{N}$

$\mathrm{C}_{\mathrm{d}} \quad$ drag coefficiet

$\mathrm{D}_{\mathrm{e}} \quad$ sphere diameter, $\mathrm{m}$

$\mathrm{F}_{\mathrm{D}}$ drag force, $\mathrm{N}$

$\mathrm{F}_{\mathrm{t}}$ resultant force in the tangential direction, $\mathrm{N}$

g gravity acceleration, $\mathrm{m} / \mathrm{s}^{2}$

1 length of the pendulum cord, $m$

$\mathrm{m}_{\mathrm{e}} \quad$ mass of the sphere, $\mathrm{Kg}$

$\mathrm{Re}_{\mathrm{D}}$ Reynolds number based on the sphere diameter

$\mathrm{T}$ tension force, $\mathrm{N}$

$\mathrm{t}$ time, $\mathrm{s}$

$\mathrm{V}$ velocity vector of the sphere, $\mathrm{m} / \mathrm{s}$

$\forall_{\mathrm{e}} \quad$ sphere volume, $\mathrm{m}^{3}$

W weight, $\mathrm{N}$

\section{Greek symbols}

$v_{f} \quad$ kinematics viscosity of the fluid, $\mathrm{m}^{2} / \mathrm{s}$

$\theta \quad$ angle, degrees

$\rho_{\mathrm{e}} \quad$ sphere density, $\mathrm{Kg} / \mathrm{m}^{3}$

$\rho_{\mathrm{f}} \quad$ fluid density, $\mathrm{Kg} / \mathrm{m}^{3}$

$\omega_{\mathrm{e}} \quad$ angular velocity of the sphere, $\mathrm{rad} / \mathrm{s}$

\section{INTRODUCTION}

The pendulum motion was first studied several centuries ago when Galileo Galilei (1564-1642) has established its periodicity. Newton has performed experiments for the oscillations of a pendulum immersed in different types of fluids and established that damping is proportional to the fluid density and viscosity (Newton, 1687).

In the present work, a theoretical and experimental analysis of the dynamic behavior of a viscously damped pendulum was developed. A spherical non rotational single-degree-of-freedom pendulum was tested experimentally in a large reservoir containing a Newtonian fluid. The mathematical model is represented by two ordinary differential equations (ODE). This model was defined by using a drag coefficient correlation and a viscous damping concept. The viscous damping constant was determined experimentally and used in the model proposed. The ordinary differential equation (ODE) was solved by using a fourth-order Runge-Kutta method (Ruggiero and Lopes, 1988).

The analysis of this simple dynamic system can be very useful to understand physical characteristics that are related to its dynamic behavior. The damping process, for instance, can be understood as being composed of two regimes: in the first, it can be seen as a viscous low Reynolds number flow and, in the second, it can be considered as a viscous damped system. As it will be seen later, the proposed combined mathematical model will take into account these two aspects. Another interesting point is that this can be considered as one of the simplest fluid structure interaction problems. As will be shown in the present paper, the flow over the spherical pendulum is very unstable, which creates strong interaction of the flow instabilities with the sphere movement. This means that this problem, in spite of its simplicity, contains important features regarding the dynamics of the system, both from the experimental and analytical viewpoints. It can be added that this problem could also be used for validating more complex fluid-structure 
configurations whose modeling are based on partial differential equations (PDE).

In the literature, many papers presenting results of analysis regarding the movement of a viscously damped pendulum are found (Stokes, 1850). A comparison between experimental and theoretical results of the pendulum motion was presented by Price (2003). In some papers, experimental results are used in the theoretical solutions, normally with a viscous term, which is added to the equation of motion of the pendulum (Thomsen, 2003). Other papers use a correlation for the drag coefficient (Nelson and Olsson, 1986) and the Stokes' flow equation (Peters, 2003). In spite of the type of damping term used, it was observed, in many papers, a tendency for obtaining linear solutions, which represents a severe simplification of the problem (Fitzpatrick, 2006). In the present contribution, the non-linearity of the system is preserved and is taken into account along the analysis.

Through the present work, the authors intend to contribute with other researchers that develop theoretical models and look for experimental results in the broad area of fluid-structure interaction. The proposed theoretical model is composed of two types of ODE. As mentioned above, the first one is based on the drag coefficient concept and the other is based on the viscous damping coefficient. The first model is applicable as far as a relative motion involving the sphere and the fluid occurs. Under this condition, the model leads to good results as compared with the experimental ones. However, the second model should be applied as the amplitude of the pendulum oscillations becomes small and the system exhibits a viscously damped behavior. In this case, the relative motion between the sphere and the fluid disappears. Consequently, this two-fold model gives a good representation of the entire experiment: in the beginning, the pendulum oscillations amplitude is large; later on, due to the damping effect, the amplitude values are reduced and tend to zero. This represents an asymptotical behavior, which is not simple to be modeled by using an ODE model, because the drag force tends to zero more rapidly than the amplitude of the pendulum oscillation.

The experimental part of the present work is based on a very simple and low cost image capturing process. A standard digital camera recorder is used together with image post-processing software. The experiment was developed inside a small glass-made water reservoir, as shown in figure 1.

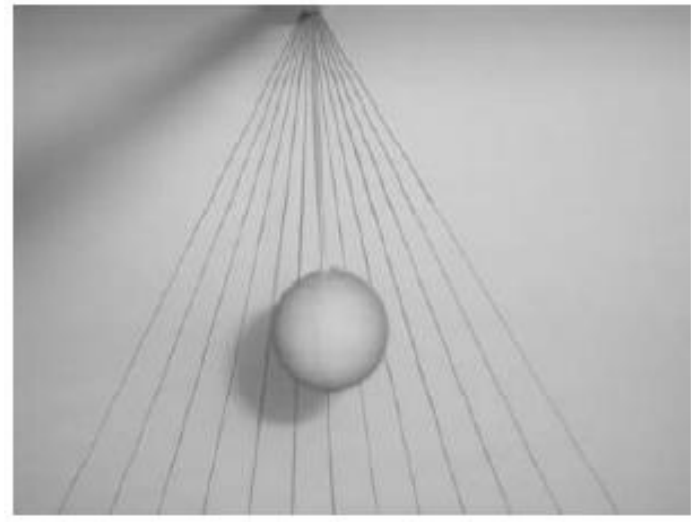

Figure 1. Water reservoir containing an immersed sphere tethered by a very fine cord.

For very small amplitudes of the oscillation $\theta(\mathrm{t})$, a viscous damping constant was determined from experimental results and was used in the mathematical model (viscous system), as will be described in the following.

\section{MATHEMATICAL MODEL AND NUMERICAL METHODS}

The mathematical model was established by considering a one-dimensional displacement without taking into-account the rotation of the sphere. The sphere displaces only along the angle $\theta$, as shown in figures 1 and 2. A drag coefficient correlation as a function of the Reynolds number was considered.

\section{MATHEMATICAL MODEL}

The mathematical model is based on the force diagram shown in Fig. 2, where $\mathrm{T}$ is the tension force, $\mathrm{F}_{\mathrm{D}}$ is the drag force, $\mathrm{W}$ is the weight, $\mathrm{B}$ is the buoyancy and $\mathrm{V}$ represents the velocity vector of the sphere. As previously mentioned, the mathematical model encompasses two phases. In the first phase the value of the amplitude corresponding to the sphere displacements is in the interval $2,5^{\circ} \leq \theta(\mathrm{t}) \leq 30^{\circ}$ and the resulting ODE takes into account the drag coefficient. For small amplitudes, $\theta(\mathrm{t})<2,5^{\circ}$, however, a second phase is performed in which the model behaves like a viscously damped system. This means that the model based on the drag coefficient does not work satisfactorily in this regime of oscillation, which is characterized by a very low Reynolds' number. 


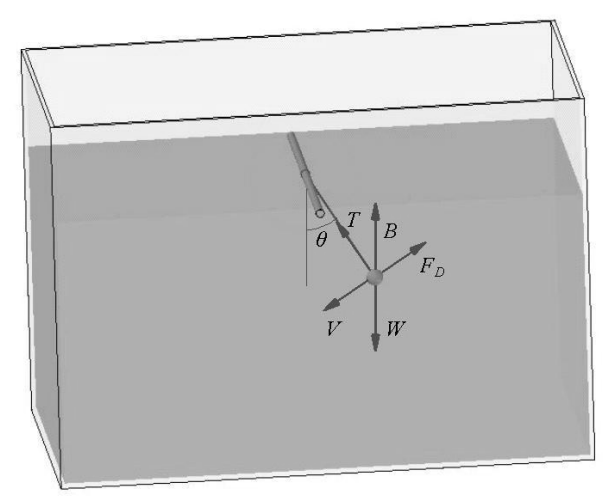

Figure 2. Force diagram for a spherical pendulum.

There are three forces acting in the tangential direction, namely, the drag force, $\mathrm{F}_{\mathrm{D}}$, and the projections of the weight and buoyancy forces on this direction. The weight, the buoyancy and the drag forces are given by Fox and McDonald (1988):

$$
\begin{aligned}
& B=\rho_{\mathrm{f}} g \nabla_{\mathrm{e}}, \\
& \mathrm{W}=\rho_{\mathrm{e}} \mathrm{g} \forall_{\mathrm{e}} \text {, } \\
& F_{D}=\frac{C_{D} \rho_{f}\left(\omega_{\mathrm{e}} 1\right)^{2} A_{p}}{2} \text {, }
\end{aligned}
$$

where $g$ is the gravity acceleration, $\rho_{\mathrm{f}}$ is the fluid density, $\forall_{\mathrm{e}}$ is the sphere volume, $\rho_{\mathrm{e}}$ is the sphere density, $\mathrm{C}_{\mathrm{D}}$ is the drag coefficient, $\omega_{\mathrm{e}}$ is the angular velocity of the sphere, 1 is the length of the pendulum cord, $\mathrm{A}_{\mathrm{p}}=\pi \mathrm{D}_{\mathrm{e}}^{2} / 4$ is the area of the sphere projected on a plane normal to the direction of its displacement and $\mathrm{D}_{\mathrm{e}}$ is the sphere diameter.

The drag coefficient can be calculated by using an experimental correlation as presented by White (1991). This correlation is given by:

$$
C_{d}=\frac{24 v_{f}}{\omega_{\mathrm{e}} 1 \mathrm{D}_{\mathrm{e}}}+\frac{6}{1+\sqrt{\mathrm{Re}_{\mathrm{D}}}}+0.4
$$

where $v_{f}$ is the kinematics viscosity of the fluid and $\operatorname{Re}_{\mathrm{D}}$ is the Reynolds number (based on the sphere diameter) given by $\operatorname{Re}_{\mathrm{D}}=\frac{\omega_{\mathrm{e}} 1 \mathrm{D}_{\mathrm{e}}}{v_{\mathrm{f}}}$. direction:

Newton's second law gives for the tangential

$$
\sum F_{t}=m_{e} a_{t}
$$

where $\sum \mathrm{F}_{\mathrm{t}}$ and $\mathrm{a}_{\mathrm{t}}$ are, respectively, the resultant force and the acceleration in the tangential direction, and $\mathrm{m}_{\mathrm{e}}$ is the mass of the sphere. Considering that:

$a_{t}=1\left(\frac{d^{2} \theta}{{d t^{2}}^{2}}\right)$,

and replacing Eqs. (1), (2), (3) and (6) in Eq. (5), yields:

$m_{e} 1\left(\frac{d^{2} \theta}{{d t^{2}}^{2}}\right)=(B-W) \sin \theta \pm F_{D}$.

The signal of the force $F_{D}$ changes according to the motion of the sphere. If the motion is clockwise, then the drag force is positive, otherwise it is negative.

Replacing B, W and $\mathrm{F}_{\mathrm{D}}$ in Eq. (7), and rewriting this equation one obtains:

$\frac{\mathrm{d}^{2} \theta}{\mathrm{dt}^{2}}+\alpha \sin \theta+\beta=0$,

where

$$
\begin{aligned}
& \alpha=\frac{g}{1}\left(1-\frac{\rho_{\mathrm{f}}}{\rho_{\mathrm{e}}}\right), \\
& \beta= \pm \frac{\mathrm{C}_{\mathrm{D}} \omega_{\mathrm{e}}{ }^{2} 1 \mathrm{~A}_{\mathrm{p}}}{2 \mathrm{~V}_{\mathrm{e}} \mathrm{l}} .
\end{aligned}
$$

It can be seen that $\mathrm{C}_{\mathrm{d}}$ depends on the Reynolds' number. In order to solve equation (8), $\mathrm{C}_{\mathrm{d}}(\mathrm{Re})$ given by equation (4), has to be used. The corresponding solution to the resulting equation gives the angular motion $\theta(\mathrm{t})$. Then, a simple derivation leads to:

$\omega_{\mathrm{e}}=\frac{\mathrm{d} \theta}{\mathrm{dt}}$

The initial condition is given by $\theta(0)=\theta_{0}$, which is specified at the beginning of the simulation. The numerical procedure to solve this equation will be described later on. The following results show that this model works well for the cases in which the amplitude of the pendulum displacement is greater than $2.5^{\circ}$. The error between the theoretical results and the experimental ones increase as the amplitude of $\theta(t)$ decreases.

Another type of model that the authors have studied is based on the concept of viscous damping, 
which is valid for small amplitude oscillations. Using Newton's second law and taking into account a damping force that is proportional to the velocity, the following equation is obtained (Thomsen, 2003):

$\mathrm{m}_{\mathrm{e}} \mathrm{l}\left(\frac{\mathrm{d}^{2} \theta}{\mathrm{dt}^{2}}\right)+(\mathrm{W}-\mathrm{B}) 1 \sin \theta+\mathrm{C} \frac{\mathrm{d} \theta}{\mathrm{dt}}=0$,

that can be rewritten as

$\frac{\mathrm{d}^{2} \theta}{\mathrm{dt}^{2}}+\beta \frac{\mathrm{d} \theta}{\mathrm{dt}}+\alpha \sin \theta=0$,

where

$\alpha=\frac{\mathrm{g}}{1}\left(1-\frac{\rho_{\mathrm{f}}}{\rho_{\mathrm{e}}}\right)$,

$\beta=\frac{\mathrm{C}}{\mathrm{m}_{\mathrm{e}} \mathrm{l}}$.

It can be observed that the signal of the damping term is given by the derivative $\frac{\mathrm{d} \theta}{\mathrm{dt}}$. The models given by equations (8), (9) and (10) and by equations (13), (14) and (15) give the angular displacement, $\theta(\mathrm{t})$, of the pendulum. The criterion regarding the application of these two models will be discussed in the following.

\section{NUMERICAL METHODS}

Equations (8) and (13) were solved by using an explicit fourth-order Runge-Kutta method. First, these equations are decomposed in a system of two first-order equations, as given below:

$\frac{\mathrm{d} \omega_{\mathrm{e}}}{\mathrm{dt}}=\mathrm{f}\left(\omega_{\mathrm{e}}, \theta\right)$

$\frac{\mathrm{d} \theta_{\mathrm{e}}}{\mathrm{dt}}=\omega_{\mathrm{e}}(\mathrm{t})$

where $\mathrm{f}\left(\omega_{\mathrm{e}}, \theta\right)$ is given, respectively, by equations (8) and (13), depending on the model used. This procedure leads, respectively, to equations (18) and (19).

$$
\begin{aligned}
& \mathrm{f}\left(\omega_{\mathrm{e}}, \theta\right)=-(\alpha \sin \theta+\beta), \text { if } \theta \geq 2.5^{\circ}, \\
& \mathrm{f}\left(\omega_{\mathrm{e}}, \theta\right)=-\left(\alpha \sin \theta+\beta \omega_{\mathrm{e}}\right) \text {, if } \theta_{e}<2.5^{\circ} .
\end{aligned}
$$

One must solve these two systems of equations in order to obtain simultaneously $\omega_{\mathrm{e}}(\mathrm{t})$ and $\theta(\mathrm{t})$. The fourth-order Runge-Kutta method requires the calculation of four constants for each dependent variable $\theta(\mathrm{t})$ and $\omega_{e}(t)$. These constants are named $K_{i \theta}$ and $K_{i \omega}$, with $\mathrm{i}=1,2,3,4$.

The recursive equations for $\theta(t)$ and $\omega_{e}(t)$ are written as:

$$
\begin{aligned}
& \omega_{\mathrm{p}}^{\mathrm{n}+1}=\omega_{\mathrm{p}}^{\mathrm{n}}+\frac{1}{6}\left(\mathrm{~K}_{1 \omega}+2 \mathrm{~K}_{2 \omega}+2 \mathrm{~K}_{3 \omega}+\mathrm{K}_{4 \omega}\right), \\
& \theta^{\mathrm{n}+1}=\theta^{\mathrm{n}}+\frac{1}{6}\left(\mathrm{~K}_{1 \theta}+2 \mathrm{~K}_{2 \theta}+2 \mathrm{~K}_{3 \theta}+\mathrm{K}_{4 \theta}\right),
\end{aligned}
$$

where the constants are given by:

$$
\begin{aligned}
& \mathrm{K}_{1 \omega}=\mathrm{dt} \mathrm{f}\left(\omega_{\mathrm{p}}^{\mathrm{n}}, \theta^{\mathrm{n}}\right), \\
& \mathrm{K}_{1 \theta}=\mathrm{dt}\left(\omega_{\mathrm{p}}^{\mathrm{n}}\right), \\
& \mathrm{K}_{2 \omega}=\mathrm{dt} \mathrm{f}\left(\omega_{\mathrm{p}}^{\mathrm{n}}+\frac{\mathrm{K}_{1 \omega}}{2}, \theta^{\mathrm{n}}+\frac{\mathrm{K}_{1 \theta}}{2}\right), \\
& \mathrm{K}_{2 \theta}=\mathrm{dt}\left(\omega_{\mathrm{p}}^{\mathrm{n}}+\frac{\mathrm{K}_{1 \omega}}{2}\right), \\
& \mathrm{K}_{3 \omega}=\mathrm{dt} \mathrm{f}\left(\omega_{\mathrm{p}}^{\mathrm{n}}+\frac{\mathrm{K}_{2 \omega}}{2}, \theta^{\mathrm{n}}+\frac{\mathrm{K}_{2 \theta}}{2}\right), \\
& \mathrm{K}_{3 \theta}=\mathrm{dt}\left(\omega_{\mathrm{p}}^{\mathrm{n}}+\frac{\mathrm{K}_{2 \omega}}{2}\right), \\
& \mathrm{K}_{4 \omega}=\mathrm{dt} \mathrm{f}\left(\omega_{\mathrm{p}}^{\mathrm{n}}+\mathrm{K}_{3 \omega}, \theta^{\mathrm{n}}+\mathrm{K}_{3 \theta}\right), \\
& \mathrm{K}_{4 \theta}=\mathrm{dt}\left(\omega_{\mathrm{p}}^{\mathrm{n}}+\mathrm{K}_{3 \omega}\right) .
\end{aligned}
$$

It is worth emphasizing that the representative model switches from equation (18) to equation (19) depending on the amplitude of $\theta_{\mathrm{e}}(\mathrm{t})$.

\section{RESULTS}

In this section, both numerical and experimental results will be shown and compared.

\section{NUMERICAL RESULTS}

The physical properties as well as the geometrical and operational parameters are specified in table 1. 
Table 1. Physical, geometrical and operational characteristics.

\begin{tabular}{cc}
\hline Specifications & Values of the parameters \\
\hline Initial angle & 30 degrees \\
Cord length & $0.1 \mathrm{~m}$ \\
Fluid viscosity & $1 \times 10^{-5} \mathrm{~m}^{2} / \mathrm{s}$ \\
Fluid density & $1,000 \mathrm{~kg} / \mathrm{m}^{3}$ \\
Sphere density & $5,565.3 \mathrm{~kg} / \mathrm{m}^{3}$ \\
Sphere diameter & $0.02198 \mathrm{~m}$ \\
\hline
\end{tabular}

Figure 3 shows the time evolution of the angular position $\theta(\mathrm{t})$. The amplitude of $\theta(\mathrm{t})$ oscillations decreases as the time increases. The displacement presents an asymptotical behavior. This figure was obtained by using the two models represented by equations (8) and (13), respectively. It is worth remembering that the model given by eq. (8) is based on the drag force concept and the model given by eq. (13) is based on the viscous damping effect. The time for which one switches from one model to the other will be discussed in the sequence of this paper. Figure 4 shows the angular velocity evolution along time, which presents a similar behavior as compared with $\theta(\mathrm{t})$.

Figure 5 presents the behavior of the Reynolds' number as a function of time. This parameter assumes values that are high enough to the flow to become transitional to turbulence. The drag force distribution is shown in figure 6 (it tends to zero as the time increases).

Figure 7 shows the four precedent quantities together (angular positions, Reynolds' number, drag force and angular velocity). As expected, the behavior of these responses is coherent and physically consistent. When $\theta$ passes through zero, the drag force, the Reynolds' number and the angular velocity, they all reach their maximum values. On the other hand, when $\theta$ reach its maximum value these quantities are all equal to zero.

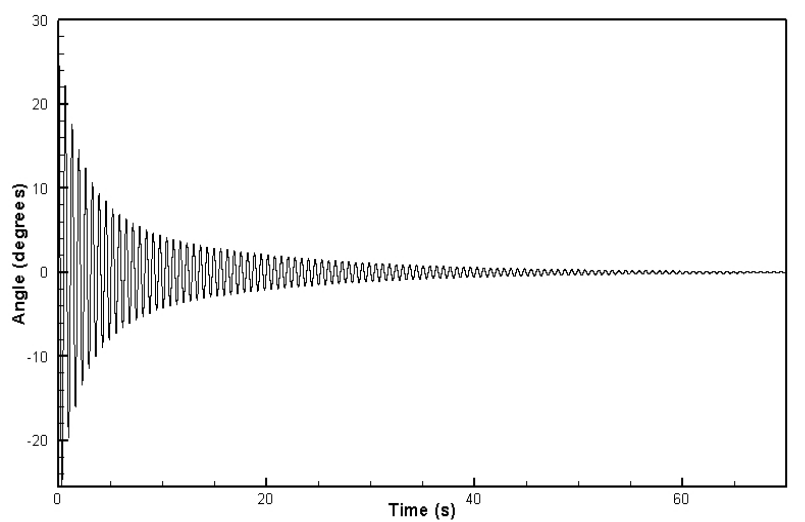

Figure 3. Time response of the angular position of the pendulum.

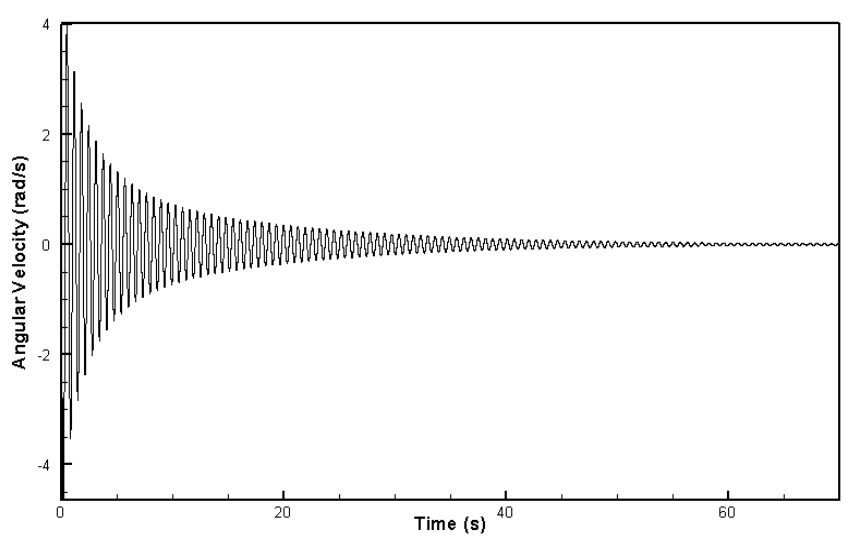

Figure 4. Time response of the angular velocity.

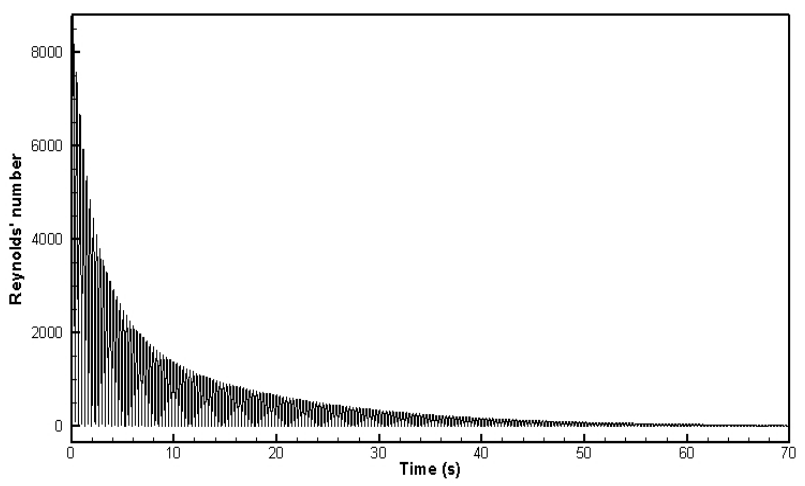

Figure 5. Time response for the Reynolds' number.

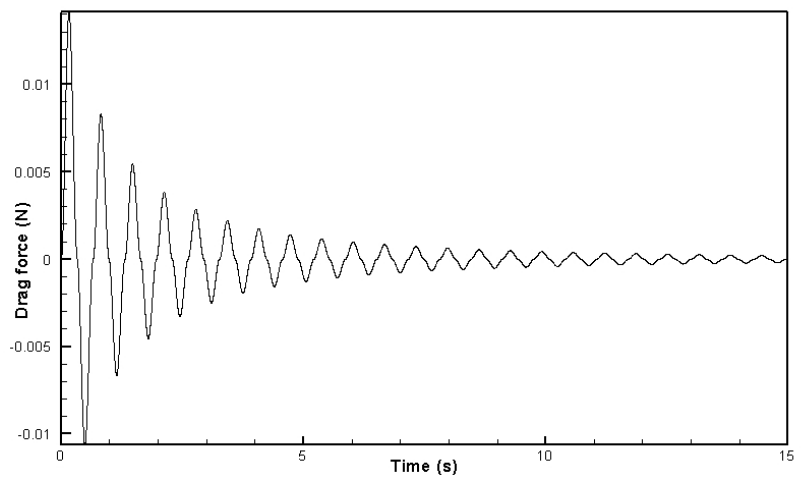

Figure 6. Time response of the drag force.

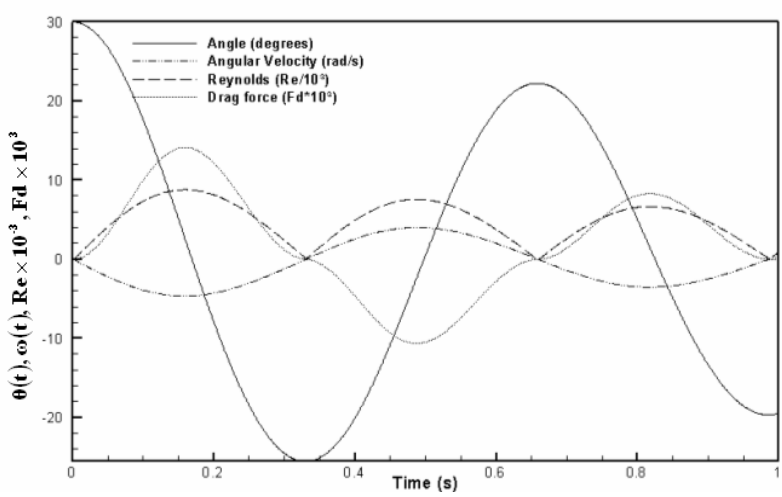

Figure 7. Time history regarding: the oscillation angle, the Reynolds' number, the drag force and the angular velocity. 


\section{EXPERIMENTAL RESULTS}

In this session, both the visualization and the experimental measurements obtained for the phenomenon studied will be shown.

\section{VISUALIZATION}

The experiment was developed using a glass reservoir partially filled with water, as illustrated in figure 8 . The dimensions of the reservoir are: $640 \mathrm{x}$ $350 \times 350 \mathrm{~cm}$.

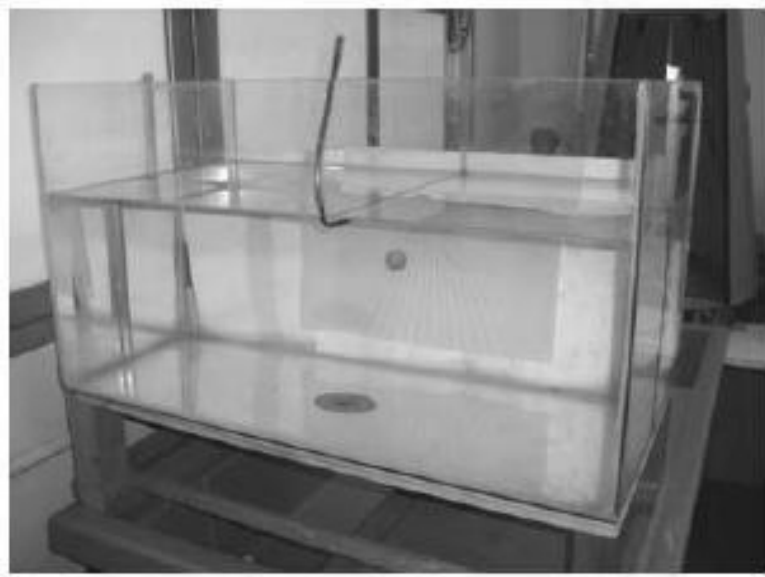

Figure 8. Glass reservoir filled with water.

The sphere was attached to a fixed frame by a $0.35 \mathrm{~mm}$ diameter nylon cord. The sphere was made of steel, with $21.98 \mathrm{~mm}$ of diameter. It can be considered that the sphere moves only in the $\theta$ direction. A sheet of paper with a scale for the angle $\theta$ was placed on the backside of the reservoir, as illustrated in figure 1 (it can also be seen in the figure 8).

In order to obtain the time response of the sphere angular position, $\theta(\mathrm{t})$, a digital camera was used. In this way, it was possible to register the images of the moving sphere and its corresponding traveling time.

To visualize qualitatively the fluid flow around the sphere, it was first embedded in red ink. Then, the sphere was carefully placed at its initial position in the reservoir and released from that position. The visualization of the fluid flow is shown in figure 9 . This visualization shows that the flow is turbulent and that the sphere passes periodically through its own unstable stream. This flow is very different from the one that happens around a stationary sphere where the flow upwind may be statistically permanent. The eq. (4) was established for this type of situation. Table 2 gives the experimental set-up values. The pendulum time response, $\theta(t)$, will be presented in the next section.
Table 2. Experimental set-up.

\begin{tabular}{cc}
\hline Specifications & Values of the parameters \\
\hline Initial angle & 30 degrees \\
Cord length & $0.086-\mathrm{m}$ \\
Fluid viscosity & $1.0 \times 10^{-5} \mathrm{~m}^{2} / \mathrm{s}$ \\
Fluid density & $1,025.25 \mathrm{~kg} / \mathrm{m}^{3}$ \\
Sphere density & $5,565.3 \mathrm{~kg} / \mathrm{m}^{3}$ \\
Sphere diameter & $0.02198 \mathrm{~m}$ \\
\hline
\end{tabular}

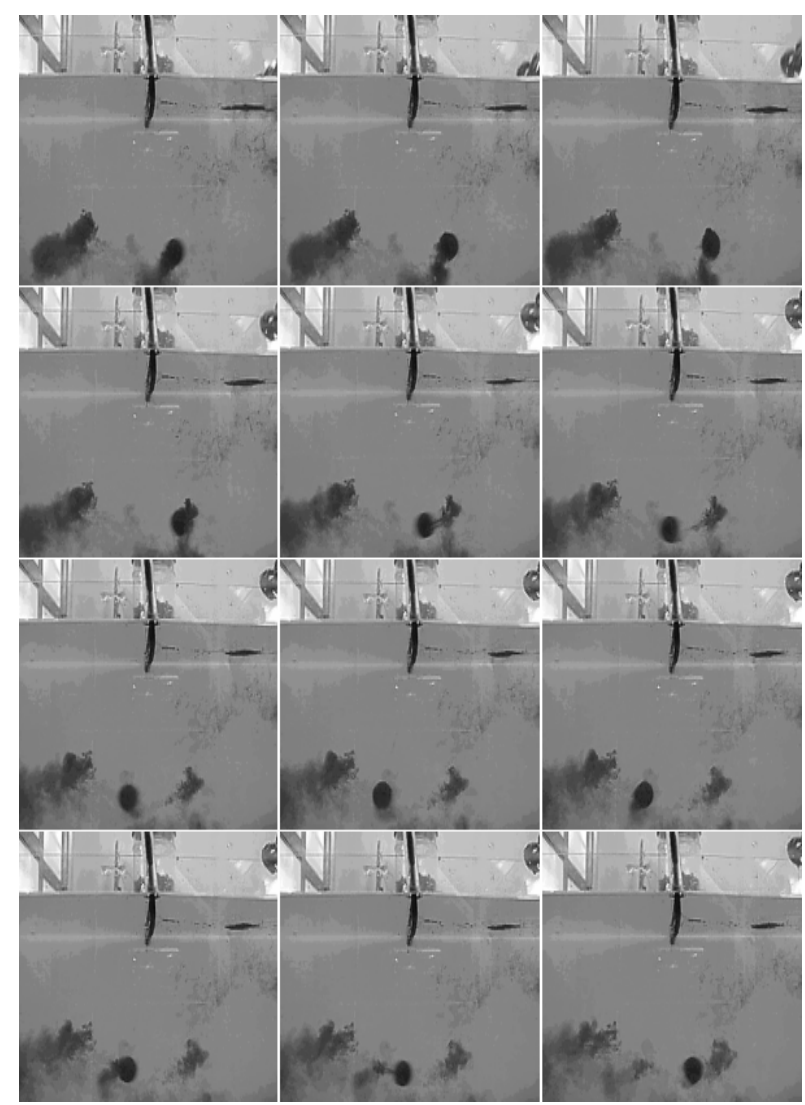

Figure 9. Visualization of the flow created by the sphere motion

\section{EXPERIMENTAL MEASUREMENTS}

The experimental measurement was obtained while the amplitude of $\theta(\mathrm{t})$ was larger than $2.5^{\circ}$ (no accuracy could be granted for smaller angles). After this point the images recorded were used only to determine the time for which the pendulum attains the rest position. Table 3 shows the experimental data corresponding to the evolution of the pendulum oscillation cycle, angle $(\theta)$, and time (s). It is possible to observe that, as expected, the amplitude of the oscillation decreases with time. It is worth mentioning that this table presents data that can be useful as a reference for validating theoretical results. 
Table 3. Experimental amplitudes.

\begin{tabular}{|c|c|c|c|c|c|}
\hline Cycle & $\begin{array}{c}\text { Angle } \\
\left({ }^{\circ}\right)\end{array}$ & Time (s) & Cycle & $\begin{array}{c}\text { Angle } \\
\left({ }^{\circ}\right)\end{array}$ & Time (s) \\
\hline 0 & 30 & 0 & 13 & 5.600 & 8.58 \\
\hline 0.5 & -25.250 & 0.33 & 13.5 & -5.350 & 8.91 \\
\hline 1 & 21.750 & 0.66 & 14 & 5.200 & 9.24 \\
\hline 1.5 & -19.125 & 0.99 & 14.5 & -5.075 & 9.57 \\
\hline 2 & 17.550 & 1.32 & 15 & 4.987 & 9.9 \\
\hline 2.5 & -16.75 & 1.65 & 15.5 & -4.887 & 10.23 \\
\hline 3 & 15.500 & 1.98 & 16 & 4.800 & 10.56 \\
\hline 3.5 & -14.250 & 2.31 & 16.5 & -4.637 & 10.89 \\
\hline 4 & 12.875 & 2.64 & 17 & 4.475 & 11.22 \\
\hline 4.5 & -12.250 & 2.97 & 17.5 & -4.325 & 11.55 \\
\hline 5 & 11.700 & 3.3 & 18 & 4.200 & 11.88 \\
\hline 5.5 & -10.875 & 3.63 & 18.5 & -4.050 & 12.21 \\
\hline 6 & 10.375 & 3.96 & 19 & 3.825 & 12.54 \\
\hline 6.5 & -9.875 & 4.29 & 19.5 & -3.650 & 12.87 \\
\hline 7 & 9.375 & 4.62 & 20 & 3.425 & 13.2 \\
\hline 7.5 & -8.625 & 4.95 & 20.5 & -3.225 & 13.53 \\
\hline 8 & 8.075 & 5.28 & 21 & 3.000 & 13.86 \\
\hline 8.5 & -7.750 & 5.61 & 21.5 & -2.800 & 14.19 \\
\hline 9 & 7.550 & 5.94 & 22 & 2.672 & 14.52 \\
\hline 9.5 & -7.350 & 6.27 & 22.5 & -2.632 & 14.85 \\
\hline 10 & 7.175 & 6.6 & 23 & 2.585 & 15.18 \\
\hline 10.5 & -6.925 & 6.93 & 23.5 & -2.566 & 15.51 \\
\hline 11 & 6.675 & 7.26 & 24 & 2.550 & 15.84 \\
\hline 11.5 & -6.475 & 7.59 & 24.5 & -2.533 & 16.17 \\
\hline 12 & 6.200 & 7.92 & 25 & 2.520 & 16.5 \\
\hline 12.5 & -5.925 & 8.25 & 25.5 & -2.515 & 16.83 \\
\hline
\end{tabular}

Figure 10 shows a comparison between theoretical and experimental results. The experimental curve was obtained as a sequence of discrete experimental amplitudes. The theoretical curve shows numerical simulation results. For the theoretical solution, the simulation starts using the drag force concept model, which is valid as far as the error between numerical and experimental results is smaller than $1 \%$, as illustrated in figure 11 . Then, it is necessary to shift to the viscous-damped model, as the first one becomes then inappropriate to represent the dynamic behavior of the system, accordingly. The authors have chosen this criterion by considering the asymptotical behavior of the pendulum dynamics.

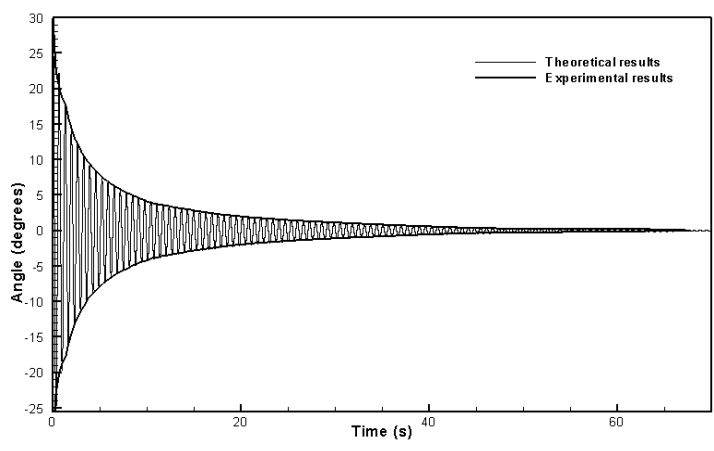

Figure 10. Comparison between theoretical and experimental results.

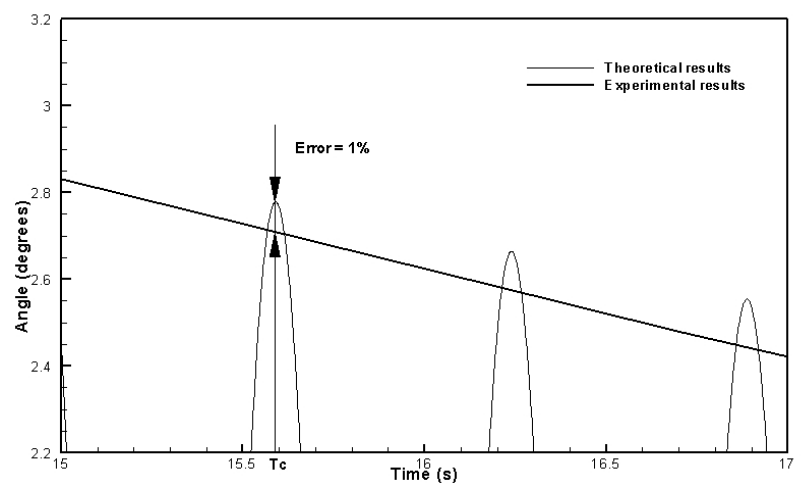

Figure 11. Time for which the error between theoretical and experimental results is smaller than $1 \%$.

\section{CONCLUSION}

In the present work, the dynamics of the classical pendulum inserted in a water reservoir was modeled theoretically and a simple experiment was performed for validation purposes. The methodology presented aims at representing the fluid-structure interaction behavior of a spherical pendulum. The proposed theoretical model is composed of two types of ordinary differential equations: the first one is based on the drag coefficient concept and the other is based on the viscous damping coefficient. The drag force based model is applicable as far as a relative motion involving the sphere and the fluid occurs. Under this condition, the model leads to good results as compared with the experimental ones. However, the second model was applied as the amplitude of the pendulum oscillations became small and the system started to exhibit a viscously damped behavior. Consequently, this two-fold model was able to provide a good representation of the dynamic behavior of the system.

The theoretical angular position of the pendulum was compared with experimental results showing very satisfactory agreement. The difference between simulation and experimental results was smaller than $1.0 \%$ for the amplitude values of the pendulum 
oscillation. Also, an error of $1.53 \%$ was found for the period of the pendulum oscillation.

Future research effort will continue in the sense of performing preliminary validation of computer fluid dynamic models, such those obtained from the immersed boundary methodology (Lima e Silva et al, 2003), by using the present research work as a reference.

\section{ACKNOWLEDGEMENTS}

The authors would like to thank CNPq (The Brazilian Council of Research and Development) for the partial financial support for this research work.

\section{REFERENCES}

Breck, W. G., Brown, R. J. C., McCoway, J. D., 1981, Chemistry for science and engineering, McGraw-Hill Ryerson.

Canuto, C., M. Y. Hussaini, A. Quarteroni, T. A. Zang, 1986, Spectral methods in fluid dynamics, Springer.

Fitzpatrick, R., 2006, The chaotic pendulum.

Fox, R. W., McDonald, A. T., Pritchard, P. J., , 2005, Introduction to Fluid Mechanics, Wiley.

Lima e Silva, A. L. F., Damasceno, J. J. R., Silveira Neto, A., 2003, Numerical simulation of two-dimensional flows over a circular cylinder using the immersed boundary method, Journal of Computational Physics, vol. 189.

Newton, I., 1972, Philosophiae Naturalis Principia Mathematica, Harvard University Press, 3rd edition.

Nelson. R. A., and M. G. Olsson, 1986, The Pendulum--Rich physics from a simple system, Am. J. Phys., Vol 54.

Peters. R. D., 2003, Nonlinear dumping of the 'linear' pendulum.

Price, J. F., 2002, Dimensional analysis of models and data sets: Similarity solutions and scaling analysis, Am. J. Phys. Vol 71.

Ruggiero, M. A. G., and V. L. R. Lopes, 1988, Numerical Calculus: theoretical and computational aspects (in Portuguese), McGraw-Hill.

Simmons, G. F., 1995, Calculus With Analytic Geometry, McGraw-Hill Science, 2nd edition.

Stokes, G. G., 1850, On the effect of the internal friction of fluids on the motion of pendulums, Trans. of the Cambridge Phil Soc. Vol. IX.

Thomsen, J. J., 2003, Vibrations and stability, Springer, 2nd edition.

White, F. M., 1991, Viscous fluid flow, McGraw-Hill Science, 2nd edition. 